\title{
Birds caught in spider webs in Asia
}

\author{
Bruno A. Walther
}

\begin{abstract}
A recent global review of birds caught in spider webs reported only three Asian cases. Given this surprisingly low number, I made a concerted effort to obtain additional Asian cases from the literature, the internet, and field workers. I present a total of 56 Asian cases which pertain to 33 bird species. As in the global dataset, mostly small bird species were caught in spider webs, with a mean body mass of $17.5 \mathrm{~g}$ and a mean wing chord length of $73.1 \mathrm{~mm}$. Consequently, birds with a body mass $>30 \mathrm{~g}$ were very rarely caught. This Asian review corroborates the global review that smaller birds are more likely to be caught and that Nephila spiders are most likely to be the predators. Continuous monitoring of spider webs is recommended to ascertain the frequency of these events.
\end{abstract}

Keywords: Spider, Predator-prey relationships, Asia

\section{Background}

Birds may be killed by environmental factors (e.g. weather; Elkins 2004), accidents or parasites (e.g. Jennings 1961), or predators. The most important predators of birds are birds, reptiles and mammals, including humans, but, more rarely, birds are also predated upon by amphibians, fish and insects (Brooks 2012). A presumably rather rare case of death occurs when a bird gets caught in a spider web; in a global review, Brooks (2012) reviewed 68 cases of birds getting trapped and often killed in the webs of large spiders. When a bird flies into a spider web, the bird may either bounce off the web or fly right through it, or it may become entangled; once entangled, the spider may or may not wrap the bird in silk. Entangled birds may then free themselves again, or they die either due to exhaustion or spider predation, while wrapped birds invariably die unless freed by humans (for details, see Brooks 2012).

Birds should therefore always attempt to avoid collision with spider webs, while the interests of spiders may differ depending on the species. Some spider species opportunistically consume trapped birds (especially large Nephila spiders, see below) and may therefore keep their webs inconspicuous to birds. However, other spider species apparently try to avoid collisions and the consequent

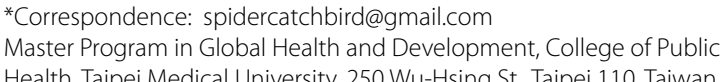

*Correspondence: spidercatchbird@gmail.com
Master Program in Global Health and Development, College of Public Health, Taipei Medical University, 250 Wu-Hsing St., Taipei 110, Taiwan, China
}

(c) 2016 The Author(s). This article is distributed under the terms of the Creative Commons Attribution 4.0 International License (http://creativecommons.org/licenses/by/4.0/), which permits unrestricted use, distribution, and reproduction in any medium, provided you give appropriate credit to the original author(s) and the source, provide a link to the Creative Commons license, and indicate if changes were made. The Creative Commons Public Domain Dedication waiver (http://creativecommons.org/ publicdomain/zero/1.0/) applies to the data made available in this article, unless otherwise stated. birds (Bruce et al. 2005; Walter and Elgar 2011).

As one may expect, Brooks' (2012) global review documented that it is almost exclusively smaller birds (mean body mass $=10.7 \mathrm{~g}$, mean wing chord length $=61 \mathrm{~mm}$ ) which get caught in spider webs. Consequently, 88 and $90 \%$ of all caught birds had a body mass $\leq 15 \mathrm{~g}$ and a wing chord length $<90 \mathrm{~mm}$, respectively. In the 34 cases in which the spider was identified, $62 \%$ belonged to the genus Nephila, and all were orb weavers except for a single Latrodectus species.

Most of these cases were reported from Africa, Australia, North America, and the Neotropics, but only a few from Europe and Asia (D. Brooks in litt. 2014). Thus, Brooks (2012) only reported three Asian cases: a Spotted Flycatcher (Muscicapa striata) in Iran (Doberski 1973), a juvenile Laughing Dove (Streptopelia senegalensis) in Oman in 2003 (Forsman 2003), and a Dusky Warbler (Phylloscopus fuscatus) in China some time before 2007 (D. Brooks in litt. 2014). Kasambe et al. (2010) presented another four cases from India not mentioned in Brooks (2012). Given that Nephila species are distributed across much of tropical, subtropical and even some parts of temperate Asia (Miyashita et al. 1998; Murphy and Murphy 2000; Lee et al. 2004; Harvey et al. 2007; Su et al. 2007, 2011; http://www.gbif.org/species/2149490), this relative lack of records seemed surprising. Therefore, I made a concerted effort to obtain additional cases of birds being caught in spider webs in Asia using various sources. 


\section{Methods}

In 2014 and early 2015, I used eight methods to obtain additional cases from the literature, the internet, Asian ornithologists, birdwatchers and birding tour leaders: (1) I emailed all the authors who published in BirdingASIA and Forktail and whose emails I could take from the journals' websites or the Web of Science. (2) I emailed all the authors of any article published in an ornithological journal listed on the Web of Science which were returned upon using the keywords "bird" and "Asia". (3) I posted requests on the birding fora of the Birds of Bangalore, Birds of Bombay, Bombay Natural History Society, Hong Kong Bird Watching Society, Hong Kong Wildlife Net, Kerala Birder, Malaysia Birders, Oriental Bird Club, Ornithological Society of the Middle East, and Pengamat Burung Indonesia. (4) I extensively used the web, images and video search functions of Google and Google Scholar using various combinations of the keywords "spider" "catch" "bird" "Asia" and names of Asian countries. (5) Upon any reply, I asked the person to forward my email request to other Asian ornithologists and birdwatchers. (6) I tried to obtain all references given in publications or websites which reported another case. (7) In early 2014, two native Chinese speakers (J.-L. Wu, T.-Y. Wu in litt. 2014) used Google Taiwan to search Taiwanese websites for cases using relevant keywords (see above), and I emailed all Taiwanese ornithologists and birders that I personally knew. (8) In late 2014, two native Japanese speakers (M. Kamioki, M. Mashiko in litt. 2014) used Google Japan to search Japanese websites for cases using relevant keywords (see above). I kindly request that further cases be reported to my email.

For easy comparison, I mirrored Brooks' (2012) analysis as much as possible. As described in Brooks (2012), I sought data on body mass and wing chord length for each bird species from various data sources (given in Table 1) and, if possible, determined the species of spider (given in Table 1). Unlike Brooks (2012), I added location and date for each record, if possible.

\section{Results}

In Asia, I was able to document 53 cases in addition to the three cases listed by Brooks (2012) (Table 1). The Asian cases now contain 33 bird species, and together with Brooks' (2012) global dataset, 84 bird species have been documented so far (Table 2). Three and 12 spider species were identified for Asia and the world, respectively; these are (with the number of Asian cases and cases from other continents in brackets): Aranens trifolium (0/1), Argiope aurantia (0/3), Argiope caphinarium (0/1), Argiope sp. (0/2), Eriophora biapicata (0/1), Latrodectes sp. (0/1), Mastophora sp. (0/1), Neoscona hentzii (0/1), Nephila antipodiana (3/3), Nephila clavipes (0/14),
Nephila pilipes (31/32), Nephila sp. (4/8), Nephilengys cruentata (0/2), Poecilotheria fasciata (1/1), and unidentified spiders (17/49) [Brooks (2012) also mentions Nephila inaurata in his text, but it is not listed in his Table 1]. Thus, 38 out of 39 identified cases (97\%) in Asia were Nephila species.

The mean body mass and mean wing chord length are slightly larger for the Asian than for the global dataset (Table 2). This difference is certainly due to the large number of hummingbirds in Brooks' (2012) dataset which are all smaller than the smallest Asian species, the Greenish Warbler (Phylloscopus trochiloides; Table 1). Means are also slightly larger for the means calculated across all species than for the means calculated across all individual cases (Table 2). This difference is due to smaller-than-average species caught repeatedly; of the 14 species with more than one case, 11 species had a body mass $\leq 10 \mathrm{~g}$ and 9 species had a wing chord length $\leq 60 \mathrm{~mm}$ (Table 1; Brooks 2012). Among the 49 Asian cases identified to bird species, 71 and $88 \%$ of all caught birds had a body mass $\leq 15 \mathrm{~g}$ and a wing chord length $<90 \mathrm{~mm}$, respectively; for the 114 global cases, the respective percentages are 82 and $89 \%$ (Table 1; Brooks 2012). A frequency diagram of all cases shows the great propensity of small-bodied birds being caught (Fig. 1). Cases with a body mass $>30$ g are exceedingly rare, and the two largest species ever caught, the Laughing Dove $(80.0 \mathrm{~g})$ and the Brown-eared Bulbul (Ixos amaurotis, $70.9 \mathrm{~g})$, are anomalies in the general trend.

The oldest case recorded in Asia is the Dusky Crag Martin (Hirundo concolor) reported in Morris (1889) that equals the previous oldest record by McCook (1889) cited in Brooks (2012). Only 11 of the Asian cases are from before 2000 (Table 1); likely reasons are that many records were reported on the internet (Table 1), and that many of the contacted ornithologists and birdwatchers were not active before 2000 .

\section{Discussion}

Birds are usually predators of spiders or the contents of their webs (e.g. Waide and Hailman 1977; Gunnarsson 2007), but when small birds encounter spider webs of large spiders, the tables can be turned. Overall, this review of Asian cases corroborates the conclusions made by Brooks (2012), namely: (1) the smaller the bird species, the higher the likelihood to be caught in spider webs; and (2) Nephila species are by far the most common spiders to catch birds in their webs.

However, my review of Asian cases suggests that cases of birds getting caught in spider webs may be as common in Asia as in other continents wherever large orb weaver spiders are common. Therefore, the small number of Asian cases in Brooks (2012) represented a biased picture 
Table 1 Birds entrapped in spider webs in Asia and their respective sizes

\begin{tabular}{|c|c|c|c|c|c|c|c|}
\hline Common name & Scientific name & Spider sp. & Mass (g) & Wing (mm) & Location & Date & Source \\
\hline Glossy Swiftlet & Collocalia esculenta & $\mathrm{Np}$ & 8.0 & $95^{0,2,3}$ & $\begin{array}{l}\text { Great Nicobar island, } \\
\text { Nicobar Islands, } \\
\text { India }\end{array}$ & Before 2010 & $\begin{array}{l}\text { Manchi and Sankaran } \\
\text { (2009) }\end{array}$ \\
\hline Edible-nest Swiftlet & Collocalia fuciphaga & S & 10.7 & $118^{1,1,3}$ & $\begin{array}{l}\text { Interview Island, } \\
\text { Andaman Islands, } \\
\text { India }\end{array}$ & June 2006 & $\begin{array}{l}\text { Manchi and Sankaran } \\
\text { (2009) }\end{array}$ \\
\hline Asian Palm Swift & Cypsiurus balasiensis & $\mathrm{Na}$ & 9.2 & $112^{1,1,0}$ & $\begin{array}{l}\text { Doi Kham, Chiang } \\
\text { Mai Province, } \\
\text { Thailand }\end{array}$ & 6 October 2014 & $\begin{array}{l}\text { W. Limparungpat- } \\
\text { thanakij in litt. } 2014\end{array}$ \\
\hline Laughing Dove & $\begin{array}{l}\text { Streptopelia senega- } \\
\text { lensis }\end{array}$ & $\mathrm{Na}$ & $80.0^{c}$ & $138^{c}$ & Oman & 〜 October 2003 & $\begin{array}{l}\text { Forsman (2003), } \\
\text { Brooks (2012), D. } \\
\text { Brooks in litt. } 2014\end{array}$ \\
\hline Pied Fantail & Rhipidura javanica & S & 12.5 & $82^{1,1,0}$ & $\begin{array}{l}\text { Near U Minh Thuong } \\
\text { National Park, Kien } \\
\text { Giang Province, } \\
\text { Vietnam }\end{array}$ & 9 August 2008 & M. Le in litt. 2014 \\
\hline $\begin{array}{l}\text { Black-naped Mon- } \\
\text { arch }\end{array}$ & Hypothymis azurea & $\mathrm{Np}$ & 11.3 & 69 & $\begin{array}{l}\text { Sanjay Gandhi } \\
\text { National Park, } \\
\text { Mumbai, India }\end{array}$ & October 1996 & Andheria $(1998,1999)$ \\
\hline Spotted Flycatcher & Muscicapa striata & S & $14.0^{c}$ & $80^{c}$ & $\begin{array}{l}\text { Main Kaleh Reserve, } \\
\text { Iran }\end{array}$ & 1972 & Doberski (1973) \\
\hline $\begin{array}{l}\text { Grey-streaked } \\
\text { Flycatcher }\end{array}$ & $\begin{array}{l}\text { Muscicapa griseis- } \\
\text { ticta }\end{array}$ & S & 15.1 & 83 & Taiwan Area & September 2008 & $\begin{array}{l}\text { http://tinyurl.com/ } \\
\text { spider-tw2 }\end{array}$ \\
\hline $\begin{array}{l}\text { Grey-streaked } \\
\text { Flycatcher }\end{array}$ & $\begin{array}{l}\text { Muscicapa griseis- } \\
\text { ticta }\end{array}$ & $\mathrm{Np}$ & 15.1 & $83^{3,5,8}$ & $\begin{array}{l}\text { Iriomote Island, } \\
\text { Japan }\end{array}$ & 5 October 2008 & $\begin{array}{l}\text { http://tinyurl.com/ } \\
\text { spider-jp6 }\end{array}$ \\
\hline $\begin{array}{l}\text { Asian Brown Fly- } \\
\text { catcher }\end{array}$ & Muscicapa dauurica & $S$ & 9.9 & $66^{1,1,0}$ & $\begin{array}{l}\text { Thap Lan National } \\
\text { Park, Nakhon } \\
\text { Ratchhasima Prov- } \\
\text { ince, Thailand }\end{array}$ & 14 November 1999 & P. Round in litt. 2014 \\
\hline $\begin{array}{l}\text { Asian Brown Fly- } \\
\text { catcher }\end{array}$ & Muscicapa dauurica & S & 9.9 & $66^{1,1,0}$ & $\begin{array}{l}\text { Po Toi Island, Hong } \\
\text { Kong, China }\end{array}$ & After 2006 & G. Welch in litt. 2014 \\
\hline $\begin{array}{l}\text { Asian Brown Fly- } \\
\text { catcher }\end{array}$ & Muscicapa dauurica & $\mathrm{Np}$ & 9.9 & $66^{1,1,0}$ & $\begin{array}{l}\text { Po Toi Island, Hong } \\
\text { Kong, China }\end{array}$ & 8 September 2011 & $\begin{array}{l}\text { M. Hale and G. Welch } \\
\text { in litt. } 2014\end{array}$ \\
\hline Hill Blue Flycatcher & Cyornis banyumas & $\mathrm{Np}$ & 14.5 & $67^{1,1,0}$ & $\begin{array}{c}\text { Bukit Larut, Perak } \\
\text { State, Malaysia }\end{array}$ & Unknown & Anonymous (1999b) \\
\hline Great Tit $^{\mathrm{a}}$ & Parus major & $\mathrm{Np}$ & 15.5 & $59^{1,0,0}$ & $\begin{array}{l}\text { Komesu, Itoman City, } \\
\text { Okinawa Island, } \\
\text { Japan }\end{array}$ & 10 August 2011 & $\begin{array}{l}\text { http://tinyurl.com/ } \\
\text { spider-jp1, http:// } \\
\text { tinyurl.com/spider- } \\
\text { jp2 }\end{array}$ \\
\hline Dusky Crag Martinº & Hirundo concolor & Pf & 13.0 & $98^{1,1,0}$ & $\begin{array}{l}\text { Shevaroys (=Ser- } \\
\text { varayan) Hills near } \\
\text { Salem, Tamil Nadu }\end{array}$ & Before 1889 & $\begin{array}{l}\text { Morris (1889) and } \\
\text { Anonymous (1999a) }\end{array}$ \\
\hline Light-vented Bulbul & Pycnonotus sinensis & $\mathrm{Np}$ & 29.7 & 85 & $\begin{array}{l}\text { Majia, Pingtung, } \\
\text { Taiwan Area }\end{array}$ & March 2004 & $\begin{array}{l}\text { http://tinyurl.com/ } \\
\text { spider-tw3 }\end{array}$ \\
\hline Styan's Bulbul & Pycnonotus taivanus & $\mathrm{Np}$ & 26.2 & 84 & $\begin{array}{l}\text { Guangfu, Hualien, } \\
\text { Taiwan Area }\end{array}$ & 18 June 2010 & $\begin{array}{l}\text { http://tinyurl.com/ } \\
\text { spider-tw4 }\end{array}$ \\
\hline $\begin{array}{l}\text { Yellow-vented } \\
\text { Bulbul }\end{array}$ & Pycnonotus goiavier & $\mathrm{Np}$ & 27.8 & $82^{1,4,6}$ & $\begin{array}{l}\text { Kledang-Sayong For- } \\
\text { est Reserve, Ipoh, } \\
\text { Perak, Malaysia }\end{array}$ & 11 February 2014 & $\begin{array}{l}\text { Amar-Singh (2014a, } \\
\text { b), Amar-Singh H. in } \\
\text { litt. } 2014\end{array}$ \\
\hline Buff-vented Bulbul & lole olivacea & S & 24.5 & $89^{1,1,0}$ & $\begin{array}{l}\text { Near Ban Bang } \\
\text { Khram, Khlong } \\
\text { Thom District, } \\
\text { Krabi (area also } \\
\text { known as Khao } \\
\text { Nor Chuchi), } \\
\text { Thailand }\end{array}$ & 7 August 2013 & P. Round in litt. 2014 \\
\hline Brown-eared Bulbul & Ixos amaurotis & $\mathrm{Np}$ & 70.9 & $116^{1,1,0}$ & $\begin{array}{l}\text { Tokunoshima Island, } \\
\text { Japan }\end{array}$ & 1 August 2010 & $\begin{array}{l}\text { http://tinyurl.com/ } \\
\text { spider-jp3 }\end{array}$ \\
\hline
\end{tabular}


Table 1 continued

\begin{tabular}{|c|c|c|c|c|c|c|c|}
\hline Common name & Scientific name & Spider sp. & Mass (g) & Wing (mm) & Location & Date & Source \\
\hline Plain Prinia & Prinia inornata & $\mathrm{Np}$ & 8.2 & 49 & $\begin{array}{l}\text { Tadoba Andhari } \\
\text { Tiger Reserve, } \\
\text { Chandrapur dis- } \\
\text { trict, Maharashtra, } \\
\text { India }\end{array}$ & October 1998 & Anonymous ( 1999a) \\
\hline Plain Prinia & Prinia inornata & $\mathrm{Np}$ & 8.2 & 49 & $\begin{array}{l}\text { Melghat Tiger } \\
\text { Reserve, northern } \\
\text { part of Amravati } \\
\text { District of Maha- } \\
\text { rashtra State, India }\end{array}$ & Before 2005 & Pande et al. (2004) \\
\hline Plain Prinia & Prinia inornata & $S$ & 8.2 & 49 & $\begin{array}{l}\text { Western Ghats, } \\
\text { Maharashtra, India }\end{array}$ & Unknown & S. Pande in litt. 2015 \\
\hline Plain Prinia & Prinia inornata & $S$ & 8.2 & 49 & Taiwan Area & September 2008 & $\begin{array}{l}\text { http://tinyurl.com/ } \\
\text { spider-tw2 }\end{array}$ \\
\hline Oriental White-eye & $\begin{array}{l}\text { Zosterops palpe- } \\
\text { brosus }\end{array}$ & $\mathrm{Na}$ & 8.6 & $51^{1,1,5}$ & $\begin{array}{l}\text { Sungei Buloh } \\
\text { Wetland Reserve, } \\
\text { Singapore }\end{array}$ & 30 April 2012 & Ong $(2012 a, b)$ \\
\hline Japanese White-eye & Zosterops japonicus & $\mathrm{Np}$ & 11.3 & 53 & $\begin{array}{l}\text { Mong Tseng Tsuen } \\
\text { (near Tsim Bei Tsui), } \\
\text { Hong Kong, China }\end{array}$ & 22 August 2004 & So (2005) \\
\hline Japanese White-eye & Zosterops japonicus & $\mathrm{Np}$ & 11.3 & 53 & $\begin{array}{l}\text { Keelung, Taiwan } \\
\text { Area }\end{array}$ & 18 August 2005 & $\begin{array}{l}\text { http://tinyurl.com/ } \\
\text { spider-tw5 }\end{array}$ \\
\hline Japanese White-eye & Zosterops japonicus & $\mathrm{Np}$ & 11.3 & 53 & Taiwan Area & Before October 2005 & $\begin{array}{l}\text { http://tinyurl.com/ } \\
\text { spider-tw7 }\end{array}$ \\
\hline Japanese White-eye & Zosterops japonicus & $\mathrm{Np}$ & 11.3 & 53 & $\begin{array}{l}\text { Badouzi, Keelung, } \\
\text { Taiwan Island }\end{array}$ & 13 August 2011 & $\begin{array}{l}\text { http://tinyurl.com/ } \\
\text { spider-tw6 }\end{array}$ \\
\hline Japanese White-eye & Zosterops japonicus & $\mathrm{Np}$ & 11.3 & 53 & $\begin{array}{l}\text { Okinawa Island, } \\
\text { Japan }\end{array}$ & November 2012 & $\begin{array}{l}\text { http://tinyurl.com/ } \\
\text { spider-jp5 }\end{array}$ \\
\hline Lanceolated Warbler & Locustella lanceolata & $S$ & 12.9 & 55 & $\begin{array}{l}\text { E-Luan-Pi lighthouse, } \\
\text { Kenting National } \\
\text { Park, Pintung } \\
\text { County, Taiwan } \\
\text { Area }\end{array}$ & 14 October 1984 & $\begin{array}{l}\text { TESRI }{ }^{\#} \text { collection } \\
\text { number w672, C.-t. } \\
\text { Yao in litt. } 2013\end{array}$ \\
\hline Grasshopper Warbler & Locustella naevia & $\mathrm{N}$ & 14.8 & 64 & $\begin{array}{l}\text { Tungareshwar } \\
\text { Wildlife Sanctuary, } \\
\text { Maharashtra, India }\end{array}$ & 18 November 2006 & Kasambe et al. (2010) \\
\hline Common Tailorbird & Orthotomus sutorius & $N$ & 7.5 & $43^{2,2,5}$ & $\begin{array}{l}\text { Mogarkasa Forest, } \\
\text { Nagpur, Maharash- } \\
\text { tra, India }\end{array}$ & 13 November 2008 & Kasambe et al. (2010) \\
\hline $\begin{array}{l}\text { Dark-necked Tai- } \\
\text { lorbird }\end{array}$ & $\begin{array}{l}\text { Orthotomus atrogu- } \\
\text { laris }\end{array}$ & $\mathrm{Np}$ & 7.7 & $38^{1,1,0}$ & $\begin{array}{l}\text { Kaeng Krachan } \\
\text { National Park, } \\
\text { Petchaburi Prov- } \\
\text { ince, Thailand }\end{array}$ & 2012 & $\begin{array}{l}\text { W. Limparungpat- } \\
\text { thanakij in litt. } 2014\end{array}$ \\
\hline Dusky Warbler & Phylloscopus fuscatus & $S$ & $11.0^{c}$ & $57^{c}$ & $\begin{array}{l}\text { Beidahe, Hebei } \\
\text { Province, China }\end{array}$ & Before 2007 & $\begin{array}{l}\text { D. Zetterström in litt. } \\
2007 \text { (D. Brooks in } \\
\text { litt. 2014) }\end{array}$ \\
\hline Arctic Warbler & Phylloscopus borealis & $\mathrm{Np}$ & 10.0 & 65 & $\begin{array}{l}\text { Yonaguni Island, } \\
\text { Japan }\end{array}$ & 10 September 2008 & $\begin{array}{l}\text { http://ameblo.jp/ } \\
\text { attacus/theme2- } \\
\text { 10004405518.html }\end{array}$ \\
\hline Arctic Warbler & Phylloscopus borealis & $\mathrm{Np}$ & 10.0 & 65 & $\begin{array}{l}\text { Bitou Cape, New Tai- } \\
\text { pei City municipal- } \\
\text { ity, Taiwan Area }\end{array}$ & 6 September 2011 & $\begin{array}{l}\text { Y.-P. Chiang in litt. } \\
\text { 2013-2014 }\end{array}$ \\
\hline Arctic Warbler & Phylloscopus borealis & $\mathrm{Np}$ & 10.0 & 65 & $\begin{array}{l}\text { Pak Sha O, Hong } \\
\text { Kong, China }\end{array}$ & 19 September 2015 & $\begin{array}{l}\text { Geoff Carey in litt. } \\
2015\end{array}$ \\
\hline Greenish Warbler & $\begin{array}{l}\text { Phylloscopus tro- } \\
\text { chiloides }\end{array}$ & $\mathrm{N}$ & 7.1 & 60 & $\begin{array}{l}\text { Bandhavgarh } \\
\text { National Park, } \\
\text { Madhya Pradesh, } \\
\text { India }\end{array}$ & 12 October 2007 & Kasambe et al. (2010) \\
\hline
\end{tabular}


Table 1 continued

\begin{tabular}{|c|c|c|c|c|c|c|c|}
\hline Common name & Scientific name & Spider sp. & Mass (g) & Wing (mm) & Location & Date & Source \\
\hline Greenish Warbler & $\begin{array}{l}\text { Phylloscopus tro- } \\
\text { chiloides }\end{array}$ & $\mathrm{N}$ & 7.1 & 60 & $\begin{array}{l}\text { Kanha National Park, } \\
\text { Madhya Pradesh, } \\
\text { India }\end{array}$ & 22 October 2008 & Kasambe et al. (2010) \\
\hline $\begin{array}{l}\text { Buff-breasted Bab- } \\
\text { bler }\end{array}$ & Pellorneum tickelli & S & 17.1 & $61^{1,1,0}$ & $\begin{array}{l}\text { Fraser's Hill, Pahang, } \\
\text { Malaysia }\end{array}$ & 5-11 June 2010 & S. Pieterse in litt. 2014 \\
\hline Taiwan Yuhina & Yuhina brunneiceps & S & 12.2 & 62 & Taiwan Area & Unknown & H.-S. Lin in litt. 2013 \\
\hline $\begin{array}{l}\text { Vinous-throated } \\
\text { Parrotbill }\end{array}$ & $\begin{array}{l}\text { Paradoxornis web- } \\
\text { bianus }\end{array}$ & $\mathrm{Np}$ & 9.3 & 52 & $\begin{array}{l}\text { Mountain Pinglin, } \\
\text { Taichung City, Taip- } \\
\text { ing District, Taiwan } \\
\text { Area }\end{array}$ & 2007 & $\begin{array}{l}\text { http://tinyurl.com/ } \\
\text { spider-tw1 }\end{array}$ \\
\hline $\begin{array}{l}\text { Brown-throated } \\
\text { Sunbird }\end{array}$ & $\begin{array}{l}\text { Anthreptes mala- } \\
\text { censis }\end{array}$ & $\mathrm{S}$ & 11.4 & $66^{1,1,3}$ & $\begin{array}{l}\text { Ipoh City, Perak, } \\
\text { Malaysia }\end{array}$ & 28 December 2007 & $\begin{array}{l}\text { Amar-Singh (2014a, } \\
\text { b), Amar-Singh H. in } \\
\text { litt. } 2014\end{array}$ \\
\hline $\begin{array}{l}\text { Eurasian Tree Spar- } \\
\text { row }\end{array}$ & Passer montanus & $\mathrm{S}$ & 23.0 & 66 & $\begin{array}{l}\text { Luku, Nantao } \\
\text { County, Taiwan } \\
\text { Area }\end{array}$ & 1990s & C.-t. Yao in litt. 2013 \\
\hline $\begin{array}{l}\text { Eurasian Tree Spar- } \\
\text { row }\end{array}$ & Passer montanus & $\mathrm{Np}$ & 23.0 & 66 & Taiwan Area & Summer 2004 & $\begin{array}{l}\text { http://tinyurl.com/ } \\
\text { spider-tw9 }\end{array}$ \\
\hline $\begin{array}{l}\text { Eurasian Tree Spar- } \\
\text { row }\end{array}$ & Passer montanus & S & 23.0 & 66 & Taiwan Area & Before August 2010 & $\begin{array}{l}\text { http://tinyurl.com/ } \\
\text { spider-tw8 }\end{array}$ \\
\hline $\begin{array}{l}\text { Eurasian Tree Spar- } \\
\text { row }\end{array}$ & Passer montanus & $\mathrm{Np}$ & 23.0 & 66 & $\begin{array}{l}\text { Jiji, Nantou County, } \\
\text { Taiwan Area }\end{array}$ & 13 August 2013 & C.-t. Yao in litt. 2013 \\
\hline $\begin{array}{l}\text { White-rumped } \\
\text { Munia }\end{array}$ & Lonchura striata & S & 11.3 & 48 & Taiwan Area & Unknown & Y.-C. Hsu in litt. 2013 \\
\hline Munia spec. & - & $\mathrm{Np}$ & - & - & $\begin{array}{l}\text { Bogor Botanical } \\
\text { garden, Bogor, } \\
\text { Indonesia }\end{array}$ & Before 1934 & Boedijn (1933) \\
\hline Munia spec. & - & $\mathrm{Np}$ & - & - & $\begin{array}{c}\text { probably near or in } \\
\text { Bogor, Indonesia }\end{array}$ & Before 1934 & Boedijn (1933) \\
\hline "Small birds" & - & $\mathrm{Np}$ & - & - & Thailand & Before 1933 & Bristowe (1932) \\
\hline Unidentified & - & $\mathrm{Np}$ & - & - & $\begin{array}{c}\text { probably near or in } \\
\text { Bogor, Indonesia }\end{array}$ & Before 1934 & Boedijn (1933) \\
\hline Unidentified & - & $\mathrm{Np}$ & - & - & $\begin{array}{l}\text { Cheung Sha, Lantau } \\
\text { Island, Hong Kong, } \\
\text { China }\end{array}$ & 8 October 2006 & Anonymous (2006) \\
\hline Unidentified & - & $\mathrm{Np}$ & - & - & $\begin{array}{l}\text { Wang Tong River, } \\
\text { Mui Wo, Lantau } \\
\text { Island, Hong Kong, } \\
\text { China }\end{array}$ & 12 October 2009 & M. Pearse in litt. 2015 \\
\hline Unidentified & - & $\mathrm{Np}$ & - & - & $\begin{array}{l}\text { Miyakojima Island, } \\
\text { Japan }\end{array}$ & 16 October 2011 & $\begin{array}{l}\text { http://tinyurl.com/ } \\
\text { spider-jp7 }\end{array}$ \\
\hline
\end{tabular}

Common and scientific bird names and taxonomic order follow Inskipp et al. (1996)

Spider species as follows: $\mathrm{S}=$ bird was caught by a spider; $\mathrm{N}=$ bird was caught by a Nephila species, family Nephilidae, suborder Araneomorphae, order Araneae; $\mathrm{Na}=$ bird was caught by Nephila antipodiana; $\mathrm{Np}=$ bird was caught by Nephila pilipes (=maculata); Pf = bird was caught by Poecilotheria (=Mygale) fasciata, family Theraphosidae, suborder Mygalomorphae, order Araneae. Body masses were obtained from Glutz von Blotzheim (1966-1996), Dunning (2008), Severinghaus et al. (2010), the Encyclopedia of Life (eol.org) and Wikipedia (en.wikipedia.org). Wing chord lengths were obtained from Glutz von Blotzheim (1966-1996) and Severinghaus et al. (2010) except when a superscript indicates the number of male, female and unsexed specimens which were measured by P. Capainolo (in litt. 2014) at the American Museum of Natural History, New York, USA, H. van Grouw (in litt. 2014) at the Natural History Museum, Tring, UK, A. Gamauf (in litt. 2014) at the Naturhistorische Museum Wien, Austria, and T. Töpfer (in litt. 2014) at the Zoologisches Forschungsmuseum Alexander Koenig, Bonn, Germany

a Also classified as Eastern Great Tit (Parus minor)

b The martin referred to in Morris (1889) must be a Dusky Crag Martin because of the record's location and the use of a house to build its nest (R. Kasambe, H. Rathore, in litt. 2014)

c I used the body masses and wing chord lengths given for the three Asian cases mentioned in Brooks (2012)

d TESRI refers to Taiwan Endemic Species Research Institute, Jiji, Nantou County, Taiwan Area 
Table 2 Mean body masses and mean wing chord lengths of birds caught in spider webs in Asia (Table 1) and the world (Table 1; Brooks 2012); naturally, cases of unidentified bird species in Table 1 were excluded

\begin{tabular}{llll}
\hline Analysis (sample size) & Spider species & Mass $(\mathbf{g})$ & Wing (mm) \\
\hline Asia & & & $68.9 \pm 20.9(38.0-138.0)$ \\
Individuals $(n=49)$ & 3 & $15.9 \pm 13.7(7.1-80.0)$ & $73.1 \pm 23.6(38.0-138.0)$ \\
Species $(n=33)$ & 3 & $17.5 \pm 16.2(7.1-80.0)$ & \\
World & 12 & $12.3 \pm 10.8(2.0-80.0)$ & $63.3 \pm 20.1(37.0-138.0)$ \\
Individuals $(n=114)$ & 12 & $13.5 \pm 11.8(2.0-80.0)$ & $66.4 \pm 21.8(37.0-138.0)$ \\
Species $(n=84)$ & &
\end{tabular}

The analyses were also split into individuals (i.e. all cases) and species (i.e. one case for each bird species). Each entry for body mass and wing chord length gives the mean \pm standard deviation and the range in brackets

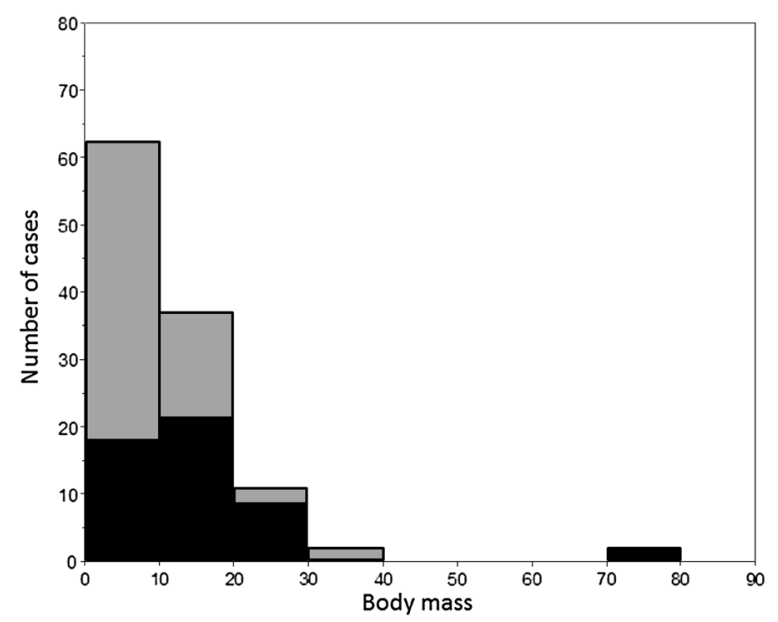

Fig. 1 Frequency diagram of body mass intervals (in steps of $10 \mathrm{~g}$ ) of 49 cases (black bars) from Asia (Table 1) and the remaining 65 cases (grey bars) from other continents (Brooks 2012), whereby each case involves one individual bird getting caught in a spider web as described in the text

of the Asian situation. Asia covers $30 \%$ of the world's terrestrial surface, and, due to this review, $46 \%$ (56 out of 121) of all documented cases now come from Asia, thus giving a more representative picture.

Naturally, reporting bias is likely to be considerable for rare natural history events like these, and Brooks (2012) therefore emphasized "the importance of reporting interesting natural history notes and keeping good field records." An example of positive reporting bias is likely to be Taiwan. At $35,883 \mathrm{~km}^{2}$, Taiwan has only 0.08 and $0.02 \%$ of the terrestrial surface of Asia and the Earth, respectively. However, the 16 cases reported from Taiwan (Table 1) represent $29 \%$ of all Asian and $13 \%$ of all global cases. One reason may be that large spiders are certainly common in Taiwan Island, and especially in somewhat disturbed or semi-open habitats with many small gaps and openings suitable for building webs, such as the coastal forests at Bitou Cape (cf. Table 1) where a large spider web can be seen approximately every $10 \mathrm{~m}$. Accordingly, Brooks et al. (2008) and Brooks (2012) hypothesized that disturbed habitats, e.g. forests disturbed by severe storms, may see an increase in the number of large spiders in the lower strata, as possible attachment sites for webs were destroyed in the upper strata, and Taiwan is regularly subjected to devastating typhoons. Furthermore, Taiwan has a very active bird-watching community and widespread internet use, evidenced by the fact that 10 of the 16 Taiwanese cases were reported on the internet (Table 1). The internet and citizen-science can thus play an increasing role in gathering and disseminating natural history information (e.g. Sullivan et al. 2014; Lin et al. 2014).

Certainly, a bird being caught in a spider web remains a rather rare event. I never encountered such a case in several years of birdwatching in tropical and subtropical regions, and 58 out of 68 people ( $85 \%)$ who replied to my request for information also never encountered such a case. The remaining people had only encountered one case in their entire life except for Amar-Singh H., S. Pande, P. Round, G. Welch, and C.-t. Yao who each had encountered two (this does not include the multiple cases reported in the publications of Boedijn 1933; Manchi and Sankaran 2009; Kasambe et al. 2010). For any small bird, it is nevertheless a considerable risk because it carries the highest fitness cost, i.e. death Combined with the facts that some spider species attempt to make their webs more visible to birds (Bruce et al. 2005; Walter and Elgar 2011), presumably to avoid collisions and the consequent damage to their webs, and that small bats are also at risk of spider predation (Nyffeler and Knörnschild 2013), the risks of collision, entanglement or death are probably high enough to facilitate the evolution of some avoidance behaviour in small birds. Even for larger bird species, there may be fitness costs; a 142 g Hooded Butcherbird (Cracticus cassicus) had to spend several minutes to preen itself after a collision with a spider web (Brooks 2012). To even begin to evaluate the magnitude of this risk, continuous video monitoring of spider webs would be required to establish collision frequencies, or captive birds could be used in experimental settings with spider webs. 


\section{Conclusions}

This study adds to the previously presented evidence (Brooks 2012) that small birds face a risk of injury or death wherever large spiders build large spider webs. Although we can assume that these events are relatively rare compared to other risks of death (e.g. predation by hawks, snakes, or humans), what remains unknown is the frequency of these events, and thus the evolutionary pressure for the evolution of countermeasures in birds. Future studies should also elucidate if spiders carry a cost (damaged web) or a benefit (additional prey) from these events, and if their web building strategies have accordingly become adapted to account for these presumably rare events.

\section{Acknowledgements}

I acknowledge the great help I received from my sources and translators, namely Amar-Singh H.S.S., Stephen Awoyemi, Anthony Bain, Daniel Brooks, Geoff Carey, Yun-Peng Chiang, Yu-Wen Emily Dai, Martin Hale, Yu-Cheng Hsu, Masayoshi Kamioki, Raju Kasambe, Manh Hung Le, Yong Ding Li, Wich'yanan Limparungpatthanakij, Hui-Shan Lin, Ruey-Shing Lin, Miyuki Mashiko, Satish Pande, Merrin Pearse, Sander Pieterse, Himanshu Rathore, Philip Round, Richard Thomas, Bas van Balen, Geoff Welch, Martin Williams, Jian-Long Wu, Tsai-Yu Wu, Cheng-te Yao, and Barure Nirmala of the Bombay Natural History Society library, all of whom I thank profusely. I also greatly thank Nancy Greig, Mark Harvey, Peter Jäger, Matjaž Kuntner and Adalberto Santos for spider identifications, and Peter Capainolo, Anita Gamauf, Paul Sweet, Till Töpfer, Tom Trombone, and Hein van Grouw for helping to obtain measurements from bird specimens. I also thank two anonymous reviewers for insightful comments.
\end{abstract}

\section{Competing interests}

The author declares that he has no competing interests.

\section{Funding}

I acknowledge financial support from Taipei Medical University through a SEED Grant.

Received: 21 June 2016 Accepted: 20 August 2016

Published online: 19 October 2016

\section{References}

Amar-Singh HSS. Yellow-vented bulbul caught in a spider's web. 2014a. http://www.besgroup.org/2014/02/13/ yellow-vented-bulbul-caught-in-a-spiders-web/.

Amar-Singh HSS. Yellow-vented Bulbul Pycnonotus goiavier. 2014b. http://orientalbirdimages.org/search. php?Bird_ID=1685\&Bird_Image_ID $=87478 \& p=10$

Andheria AP. Web of death. Newsl Birdwatchers. 1998;38:73.

Andheria AP. Blacknaped blue flycatcher Hypothymis azurea trapped in the web of the giant wood spider Nephila maculata. J Bombay Nat Hist Soc. 1999;96:145-6.

Anonymous. A bird-catching spider. Hornbill. 1999a;July-September: 12. Anonymous. A bird-eating spider! Suara Enggang 1999b;5:24-5.

Anonymous. Spider eats bird. 2006. http://www.hkbws.org.hk/BBS/viewthread. php?tid=228\&page $=1$.

Boedijn KB. Over een vogelspin. De Tropische Natuur. 1933;22:124-5.

Bristowe WS. Insects and other invertebrates for human consumption in Siam. Trans R Entomol Soc Lond. 1932:80:87-404

Brooks DM, Burkett W, Sarkozi DL. Swainson's Thrush (Catharus ustulatus) caught in web of golden silk orb weaver (Nephila clavipes). Bull Texas Ornith Soc. 2008;41:33-4.

Brooks DM. Birds caught in spider webs: a synthesis of patterns. Wilson J Ornithol. 2012;124:345-53.
Bruce MJ, Heiling AM, Herberstein ME. Spider signals: are web decorations visible to birds and bees? Biol Lett. 2005;1:299-302.

Doberski JW. Spotted Flycatcher entangled in spider's web. Brit Birds. 1973;66:401.

Dunning JB, editor. CRC Handbook of avian body masses. 2nd ed. Boca Raton, FL: CRC Press, Taylor and Francis Group; 2008.

Elkins N. Weather and bird behaviour. 3rd ed. London: T \& A D Poyser; 2004.

Forsman D. Spider catches dove: Birdvision. 2003. https://www.youtube.com/ watch?v=3Tb8-gVl1y0.

Glutz von Blotzheim UN, ed. Handbuch der Vögel Mitteleuropas (volumes 1-14). Wiesbaden, Germany: Aula-Verlag. 1966-1996.

Gunnarsson B. Bird predation on spiders: ecological mechanisms and evolutionary consequences. J Arachnol. 2007;35:509-29.

Harvey MS, Austin AD, Adams M. The systematics and biology of the spider genus Nephila (Araneae: Nephilidae) in the Australasian region. Invertebr Syst. 2007;21:407-51.

Inskipp T, Lindsey N, Duckworth W. An annotated checklist of the birds of the Oriental region. Sandy, Bedfordshire: Oriental Bird Club; 1996.

Jennings AR. An analysis of 1,000 deaths in wild birds. Bird Study. 1961;8:25-31.

Kasambe R, Thosar G, Rathore H, Shivkar A, Sasi D. Recent records of birds trapped in spiders' webs in India. BirdingASIA. 2010;13:82-4.

Lee J-W, Jiang L, Su Y-C, Tso I-M. Is Central Mountain Range a geographic barrier to the giant wood spider Nephila pilipes (Araneae: Tetragnathidae) in Taiwan? A population genetic approach. Zool Stud. 2004;43:112-22.

Lin T-E, Lin D-L, Chan, S-W, Yao C-t, Huang Z-N, Deng D-P, Mai G-S, Hsu C-H. Using Facebook community as a citizen science platform to monitor wildlife roadkill events in Taiwan (poster). 2014. Rikkyo University, Tokyo, Japan: 26th International Ornithological Congress. www.facebook.com/ groups/roadkilled/.

Manchi S, Sankaran R. Predators of swiftlets and their nests in the Andaman \& Nicobar Islands. Indian Birds. 2009;5:118-20.

McCook HC. American spiders and their spinningwork: a natural history of the orbweaving spiders of the United States, with special regard to their industry and habits. Philadelphia, PA: Academy of Natural Sciences of Philadelphia; 1889.

Miyashita T, Shinkai A, Chida T. The effects of forest fragmentation on web spider communities in urban areas. Biol Conserv. 1998;86:357-64.

Morris AW. A bird-catching spider. J Bombay Nat Hist Soc. 1889;4:69-70.

Murphy F, Murphy J. An introduction to the spiders of Southeast Asia. Kuala Lumpur: Malaysian Nature Society; 2000.

Nyffeler M, Knörnschild M. Bat predation by spiders. PLoS One. 2013;8:e58120.

Ong EL. 2012a. A white-eye entangled in a spider's web. http://www.besgroup.org/2012/04/22/a-white-eye-entangled-in-a-spiders-web/.

Ong EL. Oriental white eye trapped in spider web. 2012b. https://www.youtube.com/watch? $\mathrm{v}=$ WsG605videw.

Pande S, Tambe S, Francis MC, Sant N. Birds of Western Ghats, Kokan and Malabar (including birds of Goa). Oxford: Oxford University Press; 2004.

Severinghaus LL, Ding T-S, Fang W-H, Lin W-H, Tsai M-C, Yen C-W. The avifauna of Taiwan. Taipei, Taiwan: Forestry Bureau of Council of Agriculture of Executive Yuan; 2010 (in Chinese).

So S. Wild corner. Porcupine! 2005;32:23-25.

Su Y-C, Chang Y-H, Lee S-C, Tso IM. Phylogeography of the giant wood spider (Nephila pilipes, Araneae) from Asian-Australian regions. J Biogeogr. 2007;34:177-91.

Su Y-C, Chang Y-H, Smith D, Zhu M-S, Kuntner M, Tso I-M. Biogeography and speciation patterns of the golden orb spider genus Nephila (Araneae: Nephilidae) in Asia. Zool Sci. 2011;28:47-55.

Sullivan BL, Aycrigg JL, Barry JH, Bonney RE, Bruns N, Cooper CB, Damoulas T, Dhondt AA, Dietterich T, Farnsworth A, Fink D, Fitzpatrick JW, Fredericks T, Gerbracht J, Gomes C, Hochachka WM, Iliff MJ, Lagoze C, La Sorte FA, Merrifield M, Morris W, Phillips TB, Reynolds M, Rodewald AD, Rosenberg KV, Trautmann NM, Wiggins A, Winkler DW, Wong W-K, Wood CL, Yu J, Kelling S. The eBird enterprise: an integrated approach to development and application of citizen science. Biol Conserv. 2014;169:31-40.

Waide RB, Hailman JP. Birds of five families feeding from spider's webs. Wilson Bull. 1977:89:345.

Walter A, Elgar MA. Signals for damage control: web decorations in Argiope keyserlingi (Araneae: Araneidae). Behav Ecol Sociobiol. 2011;65:1909-15. 